18

\title{
Плазмон-экситонное взаимодействие в планарных наноструктурах с квантовыми точками*
}

\author{
(C) Т.М. Чмерева, М.Г. Кучеренко, Д.А. Кислов, В.М. Налбандян \\ Оренбургский государственный университет, \\ 460018 Оренбург, Россия \\ e-mail: chmereva@yandex.ru
}

Поступила в редакцию 06.07.2018 г.

Теоретически исследованы режимы слабой и сильной связи квантовых точек с поверхностными плазмонами в планарной наноструктуре. Проведены расчеты скоростей безызлучательной передачи энергии от квантовой точки к проводящей подложке и дисперсионных зависимостей гибридных плазмон-экситонных состояний при различных значениях параметров рассматриваемой системы. Показано, что скорость переноса энергии при межзонном переходе электрона и при переходе квантовых точек из экситонного состояния в основное может значительно превосходить скорость излучательной рекомбинации электрона и дырки. Установлено, что при определенных условиях величина расщепления Раби может достигать $100 \mathrm{meV}$ и более.

DOI: 10.21883/OS.2018.11.46835.193-18

\section{Введение}

Плазмон-экситонное взаимодействие в различных наноструктурах, содержащих проводящие элементы, квантовые точки (КТ), молекулы органического люминофора, J-агрегаты активно исследуется в последнее время как экспериментально, так и теоретически. Это обусловлено широкими перспективами его использования при разработке оптоэлектронных устройств нового поколения. Хорошо известно [1], что данное взаимодействие может проявляться в двух видах с разными оптическими свойствами: как слабое и как сильное.

Режим сильной связи реализуется, когда взаимодействие плазмона с экситоном превосходит приводящее к дезактивации электронных возбуждений в системе взаимодействие этих квазичастиц с фотонами и фононами. В этом режиме возникают два гибридных плазмон-экситонных состояния, энергии которых в точке пересечения дисперсионных кривых невзаимодействующих плазмона и экситона отличаются друг от друга на величину, называемую расщеплением Раби. Возникновение гибридных состояний экспериментально наблюдалось в слоистых структурах, состоящих из серебряной подложки, покрытой пленкой, содержащей $J$-агрегаты цианинового красителя [2,3], молекулы родамина 6G [4] или квантовые точки CdSe [5].

Классический и квантовый подходы к описанию сильной связи между излучателями и поверхностными плазмонами изложены в работах [6-9]. В классическом подходе законы дисперсии гибридных состояний следуют из дисперсионного уравнения поверхностного плазмона, в котором диэлектрические проницаемости и металлической подложки, и среды, содержащей излучатели,

* International Conference „PCNSPA 2018 - Photonic Colloidal Nanostructures: Synthesis, Properties, and Applications", Saint Petersburg, Russia, June 4-8, 2018. являются функциями частоты. В квантовом подходе эти законы определяются уравнением Шредингера, гамильтониан которого содержит оператор плазмон-экситонного взаимодействия.

Режим слабой связи плазмонов с излучателями проявляет себя в таких хорошо известных явлениях, как поверхностно-усиленное комбинационное рассеяние света, индуцированное поверхностными плазмонами усиление молекулярной флуоресценции [10], тушение возбужденных состояний молекул и КТ проводящей поверхностью [11], изменение скорости межмолекулярного переноса энергии вблизи металлических объектов [12]. В данном режиме обычно происходит односторонний перенос энергии от экситона к плазмону или наоборот. Расчет скорости такого переноса проводят, как правило, в рамках квантово-механической теории возмущений [13].

Как указывается в работе [1], режим сильной связи может быть использован для управления скоростью и выходом химических реакций, для приготовления запутанных состояний с целью передачи квантовой информации, при разработке низкопороговых лазеров. Спектр практических приложений слабого плазмонэкситонного взаимодействия еще более широк. На явлениях, обусловленных слабой связью плазмонов и экситонов, могут базироваться принципы работы органических светоизлучающих диодов, биосенсоров, флуоресцентных микроскопов, солнечных ячеек, одноэлектронных транзисторов и других современных устройств фотоники и электроники. Для эффективной работы таких устройств необходима подробная информация о влиянии их экситонной и плазмонной составляющих друг на друга.

В настоящей работе проведено теоретическое исследование взаимодействия упорядоченных и неупорядоченных массивов сферических КТ в диэлектрической 


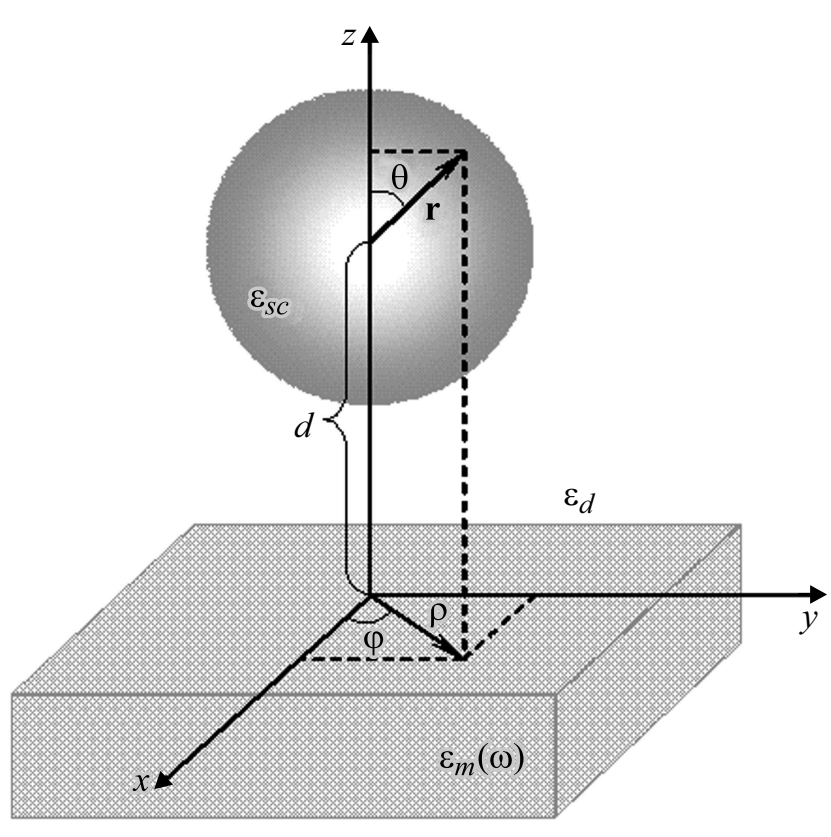

Рис. 1. Сферическая квантовая точка над плоской поверхностью металла.

среде с поверхностными плазмонами плоской металлической подложки. Для обоих видов массивов КТ рассмотрены случаи сильной и слабой связи экситонов с плазмонами. Рассчитаны скорости переноса энергии от квантовой точки в режиме сильного конфайнмента к подложке при внутри- и межзонных переходах электрона и при переходе в режиме слабого конфайнмента из экситонного в основное состояние, которые при определенных геометрических и электродинамических параметрах системы могут значительно превосходить скорость излучательной рекомбинации электрона и дырки. В классическом и квантовом подходе определены законы дисперсии гибридных плазмон-экситонных состояний для неупорядоченных массивов КТ. Для упорядоченного массива найдены скорость необратимого переноса энергии экситонного возбуждения к подложке и законы дисперсии гибридных состояний. Показано влияние параметров рассматриваемой системы на величину расщепления Раби.

\section{Релаксация электронных возбуждений КТ вблизи плоской поверхности проводника}

Возбужденная КТ, находящаяся в диэлектрической среде над металлической поверхностью (рис. 1), может перейти в основное состояние, испустив квант плазмонных колебаний. Скорость такого перехода определяется в рамках квантово-механической теории возмущений по золотому правилу Ферми, которое содержит оператор взаимодействия электрона КТ с электрическим полем поверхностного плазмона

$$
\hat{V}=-e \mathbf{r} \cdot \mathbf{E}(\boldsymbol{\rho}, z),
$$

где $\mathbf{r}$ - радиус-вектор, определяющий положение электрона, $\mathbf{E}(\boldsymbol{\rho}, z)$ - напряженность поля в месте расположения электрона.

Для КТ, находящейся в режиме сильного конфайнмента, возможна внутризонная и межзонная релаксация электронных возбуждений КТ с участием поверхностных плазмонов [14]. При внутризонной релаксации электрон совершает переходы между уровнями размерного квантования зоны проводимости, и матричный элемент оператора взаимодействия электрона КТ с полем поверхностного плазмона в дипольном приближении записывается в виде

$$
V_{f i}^{\mathrm{cc}}(\mathbf{k})=-e \int_{V_{Q D}} \psi_{n^{\prime} l^{\prime} m^{\prime}}^{*}(\mathbf{r}) \mathbf{r} \cdot \mathbf{E}_{\mathbf{k}}^{*}(\boldsymbol{\rho}, z) \psi_{n l m}(\mathbf{r}) d \mathbf{r},
$$

где $\psi_{n l m}(\mathbf{r})$ - огибающая волновая функция, являющаяся, например, волновой функцией электрона в центрально-симметричной прямоугольной яме с бесконечно высокими стенками [13], $\mathbf{E}_{\mathbf{k}}(\boldsymbol{\rho}, z)-$ компонента напряженности плазмонного поля, соответствующая волновому вектору $\mathbf{k}$, интегрирование ведется по объему КТ.

При межзонной релаксации начальное и конечное состояния электрона принадлежат разным зонам, и матричный элемент взаимодействия (1) можно представить следующим образом:

$$
V_{f i}^{\mathrm{vc}}(\mathbf{k})=-e \mathbf{r}_{\mathrm{vc}} \int_{V_{Q D}} \psi_{n^{\prime} l^{\prime} m^{\prime}}^{\mathrm{v}^{*}}(\mathbf{r}) \mathbf{E}_{\mathbf{k}}^{*}(\boldsymbol{\rho}, z) \psi_{n l m}^{\mathrm{c}} d \mathbf{r},
$$

где $e \mathbf{r}_{\mathrm{vc}}-$ дипольный матричный элемент перехода электрона из зоны проводимости в валентную зону.

Когда КТ находится в режиме слабого конфайнмента, в ней возможно образование экситонов Ванье-Мотта. В этом случае рождение поверхностного плазмона может происходить при переходе КТ из экситонного состояния в основное. При вычислении матричного элемента оператора (1) нужно использовать огибающие функции экситоноподобного состояния $\Psi\left(\mathbf{r}_{e}, \mathbf{r}_{h}\right)$, зависящие от координат узлов кристаллической решетки, которым принадлежат электрон и дырка [15]. Как известно [13], данная огибающая функция представима в виде произведения двух функций $\Phi_{n l m}(\mathbf{x}) \psi_{n^{\prime} l^{\prime} m^{\prime}}(\mathbf{X})$, первая из которых является водородоподобной функцией и описывает относительное движение электрона и дырки, вторая представляет собой функцию частицы в сферически симметричной прямоугольной потенциальной яме и описывает движение экситона как целого. Через $\mathbf{x}=\mathbf{r}_{e}-\mathbf{r}_{h}$ обозначен радиус-вектор относительного движения электрона и дырки, через $\mathbf{X}=\left(m_{e} \mathbf{r}_{e}+m_{h} \mathbf{r}_{h}\right) /\left(m_{e}+m_{h}\right)-$ радиусвектор центра масс экситона, $m_{e(h)}$ - эффективная масса электрона (дырки). При вычислении матричного элемента оператора (1) оказывается, что основной вклад 
вносит огибающая функция $\Psi(\mathbf{r}, \mathbf{r})$, в которой электрон и дырка принадлежат одной элементарной ячейке $\mathbf{r}_{e}=\mathbf{r}_{h}=\mathbf{r}$, при этом $\mathbf{x}=0, \mathbf{X}=\mathbf{r}$. Водородоподобная волновая функция, не обращающаяся в ноль в начале координат, является функцией s-состояния, в котором угловой момент относительного движения электрона и дырки равен нулю $(l=0)$. Таким образом, матричный элемент приобретает вид

$$
V_{f i}^{\mathrm{ex}}(\mathbf{k})=-e \Phi_{n 00}(0) \mathbf{r}_{\mathrm{vc}} \int_{V_{Q D}} \psi_{n^{\prime} l^{\prime} m^{\prime}}(\mathbf{r}) \mathbf{E}_{\mathbf{k}}^{*}(\boldsymbol{\rho}, z) d \mathbf{r} .
$$

В формализме вторичного квантования напряженность плазмонного поля в диэлектрической среде, примыкающей к плоской поверхности металлической подложки, имеет вид [16]

$$
\begin{gathered}
\mathbf{E}_{d}(\boldsymbol{\rho}, z)=\sum_{\mathbf{k}}\left(\mathbf{E}_{\mathbf{k}}(\boldsymbol{\rho}, z) a_{\mathbf{k}}+\mathbf{E}_{\mathbf{k}}^{*}(\boldsymbol{\rho}, z) a_{\mathbf{k}}^{+}\right), \\
\mathbf{E}_{\mathbf{k}}(\boldsymbol{\rho}, z)=\sqrt{\frac{2 \pi \hbar \omega(k)}{S L(k)}} e^{-k_{z d} z}\left(\mathbf{e}_{\mathbf{k}}+i \frac{k}{k_{z d}} \mathbf{e}_{z}\right) e^{i \mathbf{k} \boldsymbol{\rho}},
\end{gathered}
$$

где $\mathbf{e}_{\mathbf{k}}-$ единичный вектор, направленный вдоль волнового вектора $\mathbf{k}$ поверхностного плазмона; $\mathbf{e}_{z}-$ единичный вектор нормали к проводящей поверхности, площадь которой $S ; a_{\mathbf{k}}$ и $a_{\mathbf{k}}^{+}-$операторы уничтожения и рождения поверхностного плазмона с волновым вектором $\mathbf{k}, \omega(k)$ - частота поверхностного плазмона. Множитель $L(k)$ возникает при преобразовании гамильтониана электромагнитного поля поверхностного плазмона к вторично-квантованному виду, причем величина $S L(k)$ дает эффективный объем приповерхностного слоя, в котором формируются поверхностные плазмоны [16]

$$
\begin{aligned}
L(k)= & \frac{1}{2}\left\{\frac{1}{2 k_{z m}}\left[\left(1+\frac{k^{2}}{k_{z m}^{2}}\right) \frac{d\left(\omega \varepsilon_{m}\right)}{d \omega}+\frac{\omega^{2}}{c^{2}} \frac{\varepsilon_{m}^{2}}{k_{z m}^{2}}\right]\right. \\
& \left.+\frac{1}{2 k_{z d}}\left[\left(1+\frac{k^{2}}{k_{z d}^{2}}\right) \varepsilon_{d}+\frac{\omega^{2}}{c^{2}} \frac{\varepsilon_{d}^{2}}{k_{z d}^{2}}\right]\right\} .
\end{aligned}
$$

Здесь $k$ - волновое число, $k_{z m(d)}=\sqrt{k^{2}-\omega^{2} \varepsilon_{m(d)} / c^{2}}-$ величины, отвечающие за быстроту спадания напряженности поля плазмона по мере удаления от проводящей поверхности. Диэлектрическая проницаемость металлической подложки является функцией частоты и определяется в рамках обобщенной модели Друде $\varepsilon_{m}(\omega)=\varepsilon_{\infty}-\omega_{p l}^{2} / \omega^{2}$, где $\varepsilon_{\infty}$ и $\omega_{p l}$ - высокочастотная диэлектрическая постоянная и плазменная частота металла соответственно. Диэлектрическая проницаемость $\varepsilon_{d}$ среды над металлом предполагается не зависящей от частоты.

Если длина волны плазмона значительно превосходит размеры сферической КТ, то можно считать, что КТ расположена в однородном поле поверхностного плазмона. Тогда напряженность электрического поля внутри КТ связана с напряженностью поля плазмона над подложкой соотношением [17]

$$
\mathbf{E}(0, d)=\frac{\varepsilon_{d}}{\varepsilon_{\mathrm{eff}}} \mathbf{E}_{d}(0, d),
$$

где $\varepsilon_{\mathrm{eff}}=\left(\varepsilon_{s c}+2 \varepsilon_{d}\right) / 3$ - эффективная диэлектрическая проницаемость материала КТ, $\varepsilon_{s c}$ - диэлектрическая проницаемость материала КТ на частоте электронного перехода, $d$ - расстояние от поверхности металла до центра КТ.

В этом случае для скорости внутризонного перехода, сопровождающегося рождением поверхностного плазмона, получается следующее выражение:

$$
\begin{aligned}
U_{\mathrm{cc}}= & \frac{e^{2} \omega\left(k_{0}\right) k_{0} e^{-2 k_{z d} d}}{L\left(k_{0}\right) \hbar(2 l+1)}\left|\frac{d \omega}{d k}\right|_{k=k_{0}}^{-1} \frac{\varepsilon_{d}^{2}}{\varepsilon_{\mathrm{eff}}^{2}} \\
& \times \sum_{m m^{\prime} V_{Q D}} \int_{Q} d \alpha \mid \int \psi_{n^{\prime} l^{\prime} m^{\prime}}^{*}(r, \theta, \varphi)[\sin \theta \cos (\varphi-\alpha) \\
& \left.-i \cos \theta k_{0} / k_{z d}\right]\left.\psi_{n l m}(r, \theta, \varphi) r^{3} d r \sin \theta d \theta d \varphi\right|^{2},
\end{aligned}
$$

где $\alpha$ - угол, задающий направление волнового вектора поверхностного плазмона относительно оси $x$. При выводе формулы (6) дельта-функция, входящая в золотое правило Ферми и выражающая равенство частот поверхностного плазмона и соответствующего электронного перехода, была представлена в виде [13] $|d \omega / d k|_{k=k_{0}}^{-1} \delta\left(k-k_{0}\right)$, где $k_{0}$ - значение волнового числа, при котором имеет место указанное равенство. Также в формуле (6) проводится усреднение по начальным и суммирование по конечным состояниям электрона в КТ.

В предположении об однородности поля поверхностного плазмона в пределах КТ межзонные переходы будут возможны только между уровнями размерного квантования с одинаковыми квантовыми числами. Как известно, при облучении линейно поляризованным светом преимущественно возбуждаются те КТ, для которых дипольный момент перехода параллелен электрическому вектору возбуждающего излучения. Поэтому если считать, что дипольный момент перехода электрона из зоны проводимости в валентную зону $e \mathbf{r}_{\mathrm{vc}}$ составляет угол $\beta$ с нормалью к поверхности подложки, то скорость межзонного перехода, сопровождающегося рождением поверхностного плазмона, будет определяться формулой

$$
\begin{aligned}
U_{\mathrm{vc}}= & \frac{\pi e^{2} r_{\mathrm{vc}}^{2} \omega\left(k_{0}\right) k_{0} e^{-2 k_{z d} d}}{L\left(k_{0}\right) \hbar} \frac{\varepsilon_{d}^{2}}{\varepsilon_{\mathrm{eff}}^{2}} \\
& \times\left(\sin ^{2} \beta+2 \frac{k_{0}^{2}}{k_{z d}^{2}} \cos ^{2} \beta\right)\left|\frac{d \omega}{d k}\right|_{k=k_{0}}^{-1} .
\end{aligned}
$$

Подстановка в формулу (4) напряженности поля плазмона в центре КТ приводит к следующему выражению 
для скорости переноса энергии от КТ к подложке при переходе КТ из экситонного состояния в основное:

$$
\begin{aligned}
U_{\mathrm{ex}}= & \frac{\pi e^{2} r_{\mathrm{vc}}^{2} \omega\left(k_{0}\right) k_{0} \Phi_{n 00}^{2}(0) e^{-2 k_{z d} d}}{L\left(k_{0}\right) \hbar} \frac{\varepsilon_{d}^{2}}{\varepsilon_{\mathrm{eff}}^{2}}\left|\frac{d \omega}{d k}\right|_{k=k_{0}}^{-1} \\
& \times\left(\sin ^{2} \beta+2 \frac{k_{0}^{2}}{k_{z d}^{2}} \cos ^{2} \beta\right) \\
& \times\left|\int \psi_{n^{\prime} l^{\prime} m^{\prime}}(r, \theta, \varphi) r^{2} d r \sin \theta d \theta d \varphi\right|^{2}
\end{aligned}
$$

Следует заметить, что интеграл в формуле (8) отличен от нуля только, когда угловой момент экситона равен нулю $\left(l^{\prime}=0\right)$. Таким образом, взаимодействовать с поверхностными плазмонами могут только экситоны с нулевыми моментами количества движения.

В случае однородного распределения КТ в пределах пленки толщины $h$, примыкающей к плоской металлической поверхности, происходит послойное формирование кинетики тушения возбужденных состояний КТ, и временная зависимость среднеобъемной концентрации $\rho(t)$ возбужденных КТ при импульсной активации системы имеет вид

$$
\rho(t)=\frac{\rho_{0}}{h} e^{-t / \tau} \int_{R}^{h-R} e^{-U(z) t} d z,
$$

где $\tau-$ время жизни возбужденного состояния КТ; $\rho_{0}$ - концентрация возбужденных КТ в начальный момент времени, $R$ - радиус КТ, $U(z)$ - скорость релаксации, задаваемая одной из формул (6)-(8). Наблюдаемый сигнал люминесценции будет пропорционален концентрации возбуждений, определяемой выражением (9).

Как известно [18], современные технологии позволяют создавать упорядоченные массивы КТ, так называемые суперкристаллы из КТ. Вследствие взаимодействия между электронными подсистемами разных КТ возможно возникновение экситонов в суперкристалле. Авторы работы [19], используя теорию молекулярных экситонов, рассчитали энергетический спектр коллективных возбуждений электронов двумерного массива КТ с невырожденной зоной проводимости и трехкратно вырожденной валентной зоной. Авторы показали, что в таких суперкристаллах возникают три экситонные зоны, причем ширина этих зон зависит от периода решетки суперкристалла и ширины запрещенной зоны материала КТ. Так, для двумерного суперкристалла с периодом решетки $\sim 20 \mathrm{~nm}$ из КТ с узкой запрещенной зоной (например, для $\mathrm{InSb} E_{g}=0.17 \mathrm{eV}$ ) ширина экситонных зон может составлять несколько десятков $\mathrm{meV}$ [19]. $\mathrm{C}$ ростом ширины запрещенной зоны ширины экситонных зон и расстояния между ними резко уменьшаются.

В предположении узких и близкорасположенных экситонных зон скорость переноса энергии экситонного возбуждения от суперкристалла к металлической подложке будет описываться такой же формулой, что и для межзонного перехода в одиночной КТ (7). Только $k_{0}$ теперь соответствует волновому числу, при котором энергия поверхностного плазмона равна энергии перехода суперкристалла из экситонного состояния в основное. В этом случае результирующая кинетика распада населенности экситонного состояния имеет экспоненциальный вид $\rho(t)=\rho_{0} e^{-\left(U(z)+1 / \tau_{\mathrm{ex}}\right)}$, где $\tau_{\mathrm{ex}}-$ собственное время жизни экситона, т.е. в отсутствие плазмонной подсистемы.

\section{Сильная связь КТ с поверхностными плазмонами}

Когда реализуется режим сильной связи в системе, состоящей из неупорядоченного массива КТ на металлической подложке, оператор взаимодействия электронов КТ с поверхностными плазмонами $\hat{V}=-e \sum_{i} \mathbf{r}_{i} \cdot \mathbf{E}\left(\boldsymbol{\rho}_{i}, z_{i}\right)$ (суммирование по КТ) уже не будет малым, поэтому теорию возмущений использовать нельзя. Возникающие в данном случае гибридные плазмон-экситонные состояния могут быть описаны волновой функцией следующего вида [8]:

$$
\Psi=\psi_{0} \sum_{\mathbf{k}} A_{\mathbf{k}}|\mathbf{k}\rangle+\sum_{i} B_{i} \psi_{1}\left(\mathbf{r}_{i}\right)|0\rangle,
$$

где $\psi_{0}-$ волновая функция системы КТ, находящихся в основном состоянии, $\psi_{1}\left(\mathbf{r}_{i}\right)-$ волновая функция системы КТ, в которой $i$-я КТ переведена в возбужденное состояние, $|\mathbf{k}\rangle$ - волновая функция плазмонной моды, характеризуемой волновым вектором $\mathbf{k},|0\rangle-$ функция основного состояния плазмонной подсистемы. Коэффициенты $A_{\mathbf{k}}$ и $B_{i}$ определяют вклад плазмона с волновым вектором $\mathbf{k}$ и возбуждения $i$-й КТ в гибридное состояние. Эти коэффициенты можно определить из системы уравнений, которая следует из решения задачи на собственные значения $E$ оператора Гамильтона рассматриваемой системы [8]

$$
B_{i}\left(E_{\mathrm{ex}}-E\right)=e \sum_{\mathbf{k}} A(\mathbf{k}) V_{i}(\mathbf{k})
$$

$A(\mathbf{k})(\hbar \omega(k)-E)\left(E_{\mathrm{ex}}-E\right)=e^{2} \sum_{i} V_{i}^{*}(\mathbf{k}) \sum_{\mathbf{k}^{\prime}} A\left(\mathbf{k}^{\prime}\right) V_{i}\left(\mathbf{k}^{\prime}\right)$

где $V_{i}(\mathbf{k})$ - матричный элемент оператора взаимодействия электрона $i$-й КТ с полем поверхностного плазмона, $E_{\mathrm{ex}}$ - энергия возбуждения КТ, $\hbar \omega(k)-$ энергия плазмона. Как указывается в работе [8], сумма по $\mathbf{k}^{\prime}$ остается из-за отсутствия трансляционной симметрии массива КТ. Коэффициенты $A\left(\mathbf{k}^{\prime}\right)$ при $\mathbf{k}^{\prime} \neq \mathbf{k}$ отвечают за рассеяние плазмонной волны на неоднородностях среды над подложкой. Если этим рассеянием пренебречь, то из (10) получаются известные законы дисперсии гибрид- 
ных состояний [7]

$$
\begin{aligned}
E_{ \pm}(\mathbf{k})= & \frac{1}{2}\left(E_{\mathrm{ex}}+\hbar \omega(k)\right. \\
& \left. \pm \sqrt{\left(E_{\mathrm{ex}}-\hbar \omega(k)\right)^{2}+4 \sum_{i}\left|V_{i}(\mathbf{k})\right|^{2}}\right)
\end{aligned}
$$

где величина $2 \sqrt{\sum_{i}\left|V_{i}(\mathbf{k})\right|^{2}}$, вычисленная при значении волнового числа, удовлетворяющего равенству $E_{\mathrm{ex}}=\hbar \omega\left(k_{0}\right)$, есть расщепление Раби.

Если КТ распределены однородно в пределах пленки толщины $h$, то суммирование по $i$ можно заменить интегрированием $\sum_{i} \rightarrow \frac{N}{h} \int_{R}^{h-R} d z$, где $N-$ количество КТ. Так, для межзонных переходов электрона в КТ квадрат расщепления Раби определяется формулой

$$
\begin{aligned}
\Delta^{2}\left(k_{0}, \phi\right)= & \frac{4 \pi n \hbar \omega\left(k_{0}\right) e^{2} r_{\mathrm{vc}}^{2}}{L\left(k_{0}\right) k_{z d}} \frac{\varepsilon_{d}^{2}}{\varepsilon_{\mathrm{eff}}^{2}} \\
& \times\left(e^{-2 k_{z d} R}-e^{-2 k_{z d}(h-R)}\right) C(\beta, \phi),
\end{aligned}
$$

где $C(\beta, \phi)=\sin ^{2} \beta \cos ^{2} \phi+k_{0}^{2} \cos ^{2} \beta / k_{z d}^{2}, \phi-$ угол между волновым вектором плазмона и проекцией $\mathbf{r}_{\mathrm{vc}}$ на поверхность подложки, $n$ - концентрация КТ.

Как было отмечено выше, в классическом подходе [6] расщепление Раби находится из дисперсионного соотношения поверхностного плазмона $k=\frac{\omega}{c} \sqrt{\frac{\varepsilon_{m}(\omega) \varepsilon_{d}(\omega)}{\varepsilon_{m}(\omega)+\varepsilon_{d}(\omega)}}$, в котором диэлектрическая проницаемость среды, содержащей КТ, является функцией частоты и в рамках классической теории дисперсии может быть записана в виде

$$
\varepsilon_{d}(\omega)=\varepsilon_{d}^{\infty}+\frac{4 \pi n e^{2}}{m_{e}\left(\omega_{\mathrm{ex}}^{2}-\omega^{2}-i \omega \gamma\right)},
$$

где $\omega_{\mathrm{ex}}-$ частота перехода электрона в КТ, $m_{e}-$ масса электрона, $\varepsilon_{d}^{\infty}-$ высокочастотная диэлектрическая проницаемость среды, $\gamma$ - коэффициент затухания колебаний связанного электрона.

Если на поверхности металлической подложки расположен упорядоченный двумерный массив КТ, то возможно смешивание плазмонного состояния с экситонным состоянием массива. Волновая функция такого гибридного состояния будет иметь вид

$$
\Psi=\sum_{\mathbf{k}} A_{\mathbf{k}}|\mathbf{k}\rangle\left|0_{\mathrm{ex}}\right\rangle+\sum_{\mathbf{q}} B_{\mathbf{q}}|\mathbf{q}\rangle\left|0_{\mathrm{pl}}\right\rangle,
$$

где $B_{\mathbf{q}}$ - коэффициент, определяющий вклад экситона с волновым вектором q в гибридное состояние. Решение задачи на собственные значения оператора Гамильтона в этом случае приводит к равенству волновых векторов плазмона и экситона $\mathbf{k}=\mathbf{q}$ [20], и расщепление Раби будет определяться формулой

$$
\Delta^{2}\left(k_{0}, \phi\right)=\frac{8 \pi \hbar \omega\left(k_{0}\right) e^{2} r_{\mathrm{vc}}^{2} e^{-2 k_{z d} d}}{L\left(k_{0}\right) s} \frac{\varepsilon_{d}^{2}}{\varepsilon_{\mathrm{eff}}^{2}} C(\beta, \phi),
$$

где $s$ - площадь элементарной ячейки массива КТ, $d-$ расстояние от поверхности подложки до плоскости, на которой лежат центры КТ.

\section{Результаты расчетов и выводы}

При проведении расчетов скоростей релаксации КТ с участием плазмонных мод по полученным формулам (6)-(8) предполагалось, что проводящей подложкой является серебро. Энергия объемного плазмона и высокочастотная диэлектрическая проницаемость серебра принимались равными $\hbar \omega_{\mathrm{pl}}=9.1 \mathrm{eV}, \varepsilon_{\infty}=3.7$ [21]. Диэлектрическая постоянная $\varepsilon_{d}$ среды над подложкой варьировалась от 2 до 4.

В режиме сильного конфайнмента радиус экситона в объемном полупроводнике должен быть существенно больше радиуса КТ. Для КТ с радиусами $R$ меньшими $5 \mathrm{~nm}$ этому условию удовлетворяют, например, полупроводниковые КT $\mathrm{CdSe}$, для которых радиус экситонов Ванье-Мотта составляет $\sim 6 \mathrm{~nm}[22]$. В расчетах скоростей внутризонных и межзоных переходов использовались следующие характеристики объемного полупроводника: ширина запрещенной зоны $E_{g}=1.7 \mathrm{eV}$, эффективные массы электрона и дырки $m_{e}=0.13 m_{0}$ и $m_{h}=0.45 m_{0}$, где $m_{0}-$ масса свободного электрона, диэлектрическая проницаемость $\varepsilon_{\mathrm{sc}}=6$. Значение матричного элемента $\mathbf{r}_{\mathrm{vc}}$ для перехода между зоной проводимости и валентной зоной выбиралось равным $0.6 \mathrm{~nm}$.

На рис. 2 изображены зависимости скорости перехода электрона $U_{\mathrm{vc}}$ между уровнями размерного квантования,

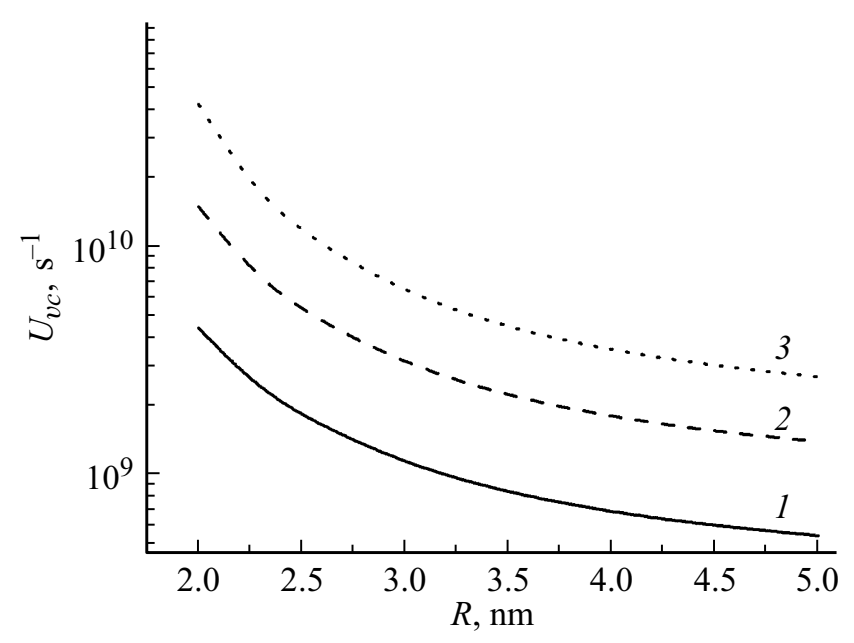

Рис. 2. Зависимость скорости межзонной релаксации от радиуса КТ при постоянном расстоянии $d=10 \mathrm{~nm}$ между центром КТ и поверхностью подложки для разных значений диэлектрической проницаемости среды над подложкой: $\varepsilon_{d}=2(1), 3$ (2), $4(3)$. 
характеризующимися квантовыми числами $n^{\prime}=1, l^{\prime}=0$ в зоне проводимости и $n=1, l=0$ в валентной зоне, от радиуса КТ при постоянном расстоянии $d=10 \mathrm{~nm}$ между центром КТ и поверхностью подложки. Представленные кривые получены в результате усреднения скорости (7) по направлениям дипольного момента перехода электрона $e \mathbf{r}_{\mathrm{vc}}$, которые предполагались равновероятными. Расчеты показывают, что зависимость квадрата модуля матричного элемента (3) $\left|V_{f i}^{\mathrm{vc}}(k)\right|^{2}$ от волнового числа плазмона является немонотонной: сначала возрастающей, затем убывающей. При этом оказывается, что в области значений $k<30 \mu \mathrm{m}^{-1}$ увеличение $\varepsilon_{d}$ приводит к уменьшению величины $\left|V_{f i}^{\mathrm{vc}}(k)\right|^{2}$, а при $k>60 \mu \mathrm{m}^{-1}$ наоборот. Скорость межзонной релаксации (7) определяется значением квадрата модуля матричного элемента в точке $k_{0}$ пересечения дисперсионной кривой $\omega(k)$ поверхностного плазмона с прямой, соответствующей частоте перехода электрона. Эта координата, в свою очередь, зависит и от электродинамических характеристик системы, и от радиуса КТ. Так, с ростом $\varepsilon_{d}$ координата $k_{0}$ смещается в область больших волновых чисел, а с ростом радиуса КТ - в обратную сторону. Кроме того, при изменении $k_{0}$ изменяется наклон дисперсионной кривой $\omega(k)$, что, как следует из формулы (7), также влияет на величину $U_{\mathrm{vc}}$. Поэтому при выбранных параметрах системы оказывается, что с ростом диэлектрической проницаемости среды над подложкой увеличивается и скорость указанного межзонного перехода, а с возрастанием радиуса КТ скорость $U_{\mathrm{vc}}$ убывает, поскольку убывает энергия межзонного перехода, стремясь к значению, характерному для объемных полупроводников.

Следует отметить, что значения волнового числа $k_{0}$ при изменении $\varepsilon_{d}$ от 2 до 4 приходятся на интервал от 15 до $40 \mu \mathrm{m}^{-1}$, при этом длина волны плазмона оказывается на два порядка больше радиуса КТ. Поэтому использование в расчетах скорости переноса энергии от КТ к подложке напряженности поля плазмона, вычисленной в центре КТ, является оправданным.

Проведенные расчеты показали, что для скоростей внутризонных переходов (6) получаются значения $10^{9} \mathrm{~s}^{-1}$, сравнимые со скоростями для других механизмов релаксации [13], только при малых радиусах КТ $R<1.5 \mathrm{~nm}$ и расстояниях между центром КТ и поверхностью подложки $d<10 \mathrm{~nm}$. С увеличением этих параметров происходит уменьшение скорости внутризонного перехода, причем с ростом радиуса КТ это уменьшение довольно резкое. Поэтому внутризонная релаксация КТ с участием поверхностных плазмонов может иметь место лишь для КТ очень малого радиуса.

Примером КТ в режиме слабого конфайнмента, служат КТ хлорида меди, которые в стеклообразной матрице имеют сферическую форму. В зависимости от условий выращивания радиус КТ $\mathrm{CuCl}$ может варьироваться в пределах от 2 до $10 \mathrm{~nm} \mathrm{[13].} \mathrm{При} \mathrm{этом}$ он будет превосходить радиус экситона Ванье-Мотта в

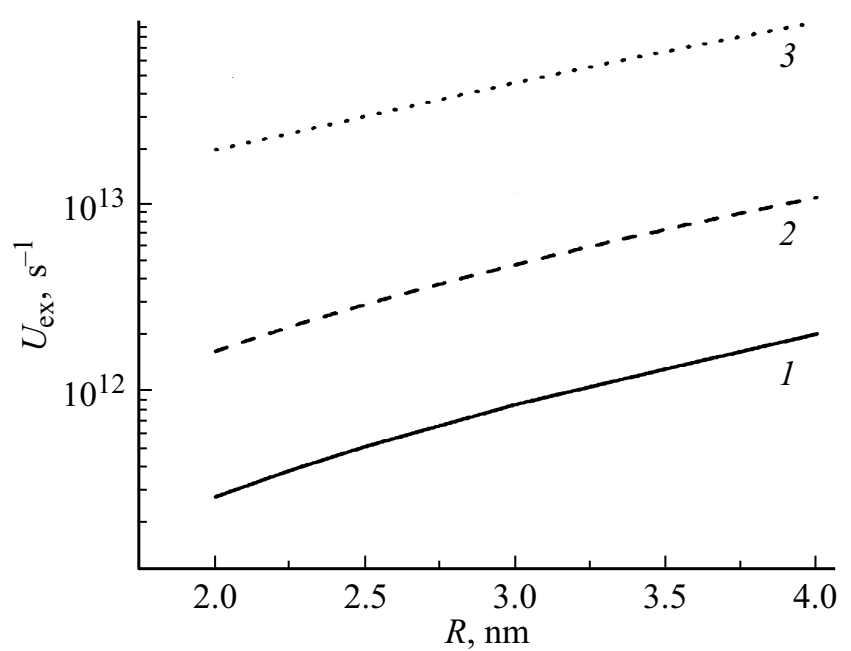

Рис. 3. Зависимость скорости перехода КТ из экситонного состояния в основное, сопровождающегося рождением поверхностного плазмона, от радиуса КТ при постоянном расстоянии $d=10 \mathrm{~nm}$. Обозначение кривых такое же, как на рис. 2.

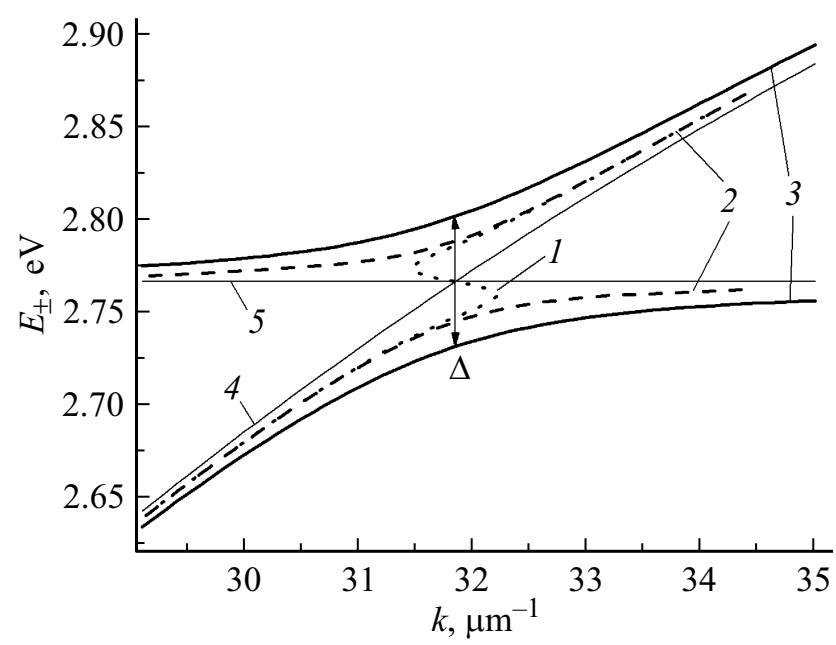

Pис. 4. Дисперсионные кривые гибридных плазмон-экситонных состояний в классическом подходе с коэффициентом затухания $\gamma=3 \cdot 10^{13} \mathrm{~s}^{-1}(1), 0$ (2) и в квантовом подходе (3); дисперсионная кривая поверхностного плазмона (4), энергия межзонного перехода (5).

объемном материале, который составляет $0.7 \mathrm{~nm}$. Хлорид меди является широкозонным полупроводником с шириной запрещенной зоны, равной $E_{g} \approx 3.3 \mathrm{eV}$. В расчетах скорости перехода КТ из экситонного состояния в основное значения статической диэлектрической проницаемости, эффективных масс электрона и дырки в объемном $\mathrm{CuCl}$ брались равными $\varepsilon_{0}=5.95, m_{e}=0.5 m_{0}$, $m_{h}=1.6 m_{0}[13]$.

Зависимости скорости $U_{\text {ex }}$ безызлучательного переноса энергии от КТ к подложке в результате перехода из экситонного состояния с квантовыми числами $n^{\prime}=1$, $l^{\prime}=0$ в основное от радиуса КТ представлены на рис. 3 . С ростом радиуса скорость $U_{\text {ex }}$ увеличивается в отличие 


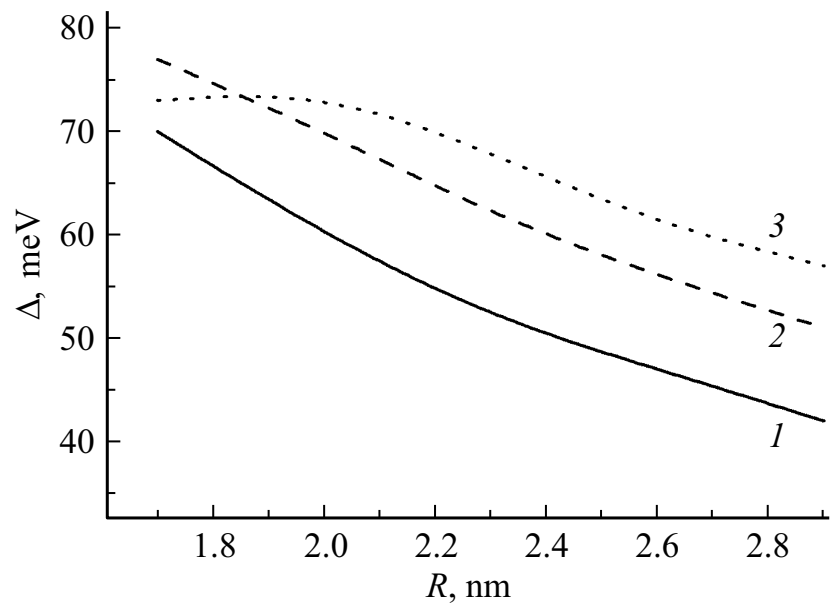

Рис. 5. Зависимость расщепления Раби от радиуса КТ. Обозначение кривых такое же, как на рис. 2.

от скоростей внутри- и межзонной релаксации, что обусловлено ростом интеграла, входящего в формулу (8).

Результаты расчетов законов дисперсии (11) гибридных плазмон-экситонных состояний для КT $\mathrm{CdSe}$ paдиуса $R=2 \mathrm{~nm}$, однородно расположенных в пленке толщиной $h=25 \mathrm{~nm}$, представлены на рис. 4 . Концентрация КТ считалась равной $n=5 \cdot 10^{18} \mathrm{~cm}^{-3}$, диэлектрическая проницаемость $\varepsilon_{d}=3$. Пунктирная кривая 1 и штриховые кривые 2 получены в классическом подходе с использованием значений коэффициента затухания $\gamma=3 \cdot 10^{13} \mathrm{~s}^{-1}$ и 0 соответственно. Сплошные кривые 3 рассчитаны в квантовом подходе. Линии 4 и 5 соответствуют дисперсионной кривой поверхностного плазмона и энергии межзонного перехода. Как показывают расчеты, увеличение коэффициента $\gamma$ приводит к уменьшению расщепления Раби и стремлению дисперсионной кривой 1 к кривой 4. При выбранных параметрах системы расщепление Раби $\Delta$, рассчитанное по формуле (12), составляет $\sim 80 \mathrm{meV}$.

Зависимость расщепления Раби от радиуса КТ при разных значениях проницаемости $\varepsilon_{d}$ представлена на рис. 5. Немонотонное поведение кривой 3 связано с тем, что при уменьшении радиуса КТ значения волнового числа $k_{0}$ становятся достаточно большими и попадают в область спадания функции $\left|V_{f i}^{\text {vc }}(k)\right|^{2}$. Отметим, что величина расщепления Раби (12) пропорциональна квадратному корню из концентрации КТ. Поэтому для КТ с достаточно малыми радиусами $(R \leq 2 \mathrm{~nm})$ возможно получение диэлектрических сред с такими концентрациями KT, которые обеспечат $\Delta>100 \mathrm{meV}$.

Таким образом, в ходе проведенного теоретического исследования установлено влияние геометрических и электродинамических параметров планарной структуры с КТ, размещенной на металлической подложке, на скорость энергетической релаксации КТ и на величину расщепления Раби. Полученные результаты согласуются с существующими экспериментальными и теоретически- ми данными $[5,7,13]$ и могут быть востребованы при разработке оптоэлектронных устройств, содержащих КТ и проводящие слои и базирующихся на сильном или слабом взаимодействии КТ с поверхностными плазмонами.

\section{Список литературы}

[1] Cao E., Lin W., Sun M., Liang W., Song Y. // Nanophotonics. 2018. V. 7. P. 145. doi 10.1515/nanoph-2017-0059

[2] Balci S., Kocabas C., Ates S., Karademir E., Salihoglu O., Aydinli A. // Phys. Rev. B. 2012. V. 86. P. 235402. doi 10.1103/PhysRevB.86.235402

[3] Bellessa J., Symonds C., Laverdant J., Benoit J.-M., Plenet J. C., Vignoli S. // Electronics. 2014. V. 3. P. 303. doi 10.3390/electronics3020303

[4] Hakala T.K., Toppari J.J., Kuzyk A., Pettersson M., Tikkanen H., Kunttu H., Torma P. // Phys. Rev. Lett. 2009. V. 103. P. 053602. doi 10.1103/PhysRevLett.103.053602

[5] Gomez D.E., Vernon K.C., Mulvaney P., Davis T.J. // Nano Lett. 2010. V. 10. P. 274. doi 10.1021/n1903455z

[6] Torma P., Barnes W.L. // Rep. Prog. Phys. 2015. V. 78. P. 013901. doi 10.1088/0034-4885/78/1/013901

[7] Gonzalez-Tudela A., Huidobro P.A., Martin-Moreno L., Tejedor C., Garcia-Vidal F.J. // Phys. Rev. Lett. 2013. V. 110. P. 126801. doi 10.1103/PhysRevLett.110.126801

[8] Agranovich V.M., Litinskaia M., Lidzey D.G. // Phys. Rev. B. 2003. V. 67. P. 085311. doi 10.1103/PhysRevB.67.085311

[9] Goliney Yu.I., Sugakov V.I., Valkunas L., Vertsimakha G.V. // Chem. Phys. 2012. V. 404. P. 116. doi 10.1016/j.chemphys.2012.03.011

[10] Dong J., Zhang Z., Zheng H., Sun M. // Nanophotonics. 2015. V. 4. P. 472. doi 10.1515/nanoph-2015-0028

[11] Akimov A.V., Mukherjee A., Yu C.L., Chang E., Zibrov A.S., Hemmer P.R., Park H., Lukin M.D. // Nature. 2007. V. 450. P. 402. doi $10.1038 /$ nature 06230

[12] Jankovski D., Bojarski P., Kwiek P., Rangelova-Jankovska S. // Chem. Phys. 2010. V. 373. P. 238. doi 10.1016/j.chemphys.2010.05.016

[13] Федоров А.В., Рухленко И.Д, Баранов А.В., Кручинин С.Ю. Оптические свойства полупроводниковых квантовых точек. СПб.: Наука, 2011. 188 с.

[14] Чмерева Т.М., Кучеренко М.Г., Дмитриев А.Д. // Опт. и спектр. 2015. T. 118. № 2. C. 300; Chmereva T.M., Kucherenko M.G., Dmitriev A.D. // Opt. Spectrosc. 2015. V. 118. N 2. P. 284. doi 10.1134/S0030400X15020058

[15] Купчак И.М., Корбутяк Д.В., Крюченко Ю.В., Саченко А.В., Соколовский И.О., Сресели О.М. // ФТП. 2006. T. 40. Вып. 1. C. 98; Kupchak I.M., Korbutyak D.V., Kryuchenko Yu.V., Sachenko A.V., Sokolovskǐ I.O., Sreseli O.M. // Semconductors. 2006. V. 40. N 1. P. 94. doi 10.1134/S1063782606010179

[16] Archambault A., Marquier F., Greffet J.-J., Arnold C. // Phys. Rev. B. 2010. V. 82. P. 035411. doi 10.1103/PhysRevB.82.035411

[17] Govorov A.O., Lee J., Kotov N.A. // Phys. Rev. B. 2007. V. 76. P. 125308. doi 10.1103/PhysRevB.76.125308

[18] Buljan M., Bogdanovic-Radovic I., Karlusic M., Desnica U.V., Radic N., Jaksic M., Salamon K., Drazic G., Bernstorff S., Holy V. // Phys. Rev. B. 2011. V. 84. P. 155312. doi 10.1103/PhysRevB.84.155312 
[19] Baimuratov A.S., Rukhlenko I.D., Fedorov A.V. // Opt. Lett. 2013. V. 38. N 13. P. 2259. doi 10.1364/OL.38.002259

[20] Чмерева Т.М., Кучеренко М.Г., Курмангалеев К.С. // Опт. и спектр. 2016. Т. 120. № 6. С. 941; Chmereva T.M., Kucherenko M.G., Kurmangaleev K.S. // Opt. Spectrosc. 2016. V. 120. N 6. P. 941. doi 10.1134/S0030400X16060060

[21] Климов В.В. Наноплазмоника. М.: Физматлит, 2009. 480 с.

[22] Карпов С.В., Микушев С.В. // ФТТ. 2010. Т. 52. Вып. 8. C. 1627; Karpov S.V., Mikushev S.V. // Physics of the solid state. 2010. V. 52. N 8. P. 1750. doi $10.1134 / \mathrm{S} 1063783410080287$ 